\title{
Association between left ventricular function and paraprotein type in patients with multiple myeloma
}

Jeong-Eun $\mathrm{Yi}^{1,2}$, Sung-Eun Lee ${ }^{2}, \mathrm{Hae}-\mathrm{Ok} \mathrm{Jung}^{2}$, Chang-Ki Min ${ }^{2}$, and Ho-Joong Youn ${ }^{2}$

${ }^{1}$ Department of Internal Medicine, Ewha Womans University Mokdong Hospital, Seoul; ${ }^{2}$ Department of Internal Medicine, College of Medicine, Seoul St. Mary's Hospital, The Catholic University of Korea, Seoul, Korea

Received: November 1, 2015

Revised : November 18, 2015

Accepted: November 18, 2015

\section{Correspondence to}

Ho-Joong Youn, M.D.

Division of Cardiology, Department of Internal Medicine, College of Medicine, Seoul St. Mary's Hospital, The Catholic University of Korea, 222 Banpo-daero, Seocho-gu, Seoul o6591, Korea

Tel: +82-2-2258-6029

Fax: +82-2-591-1506

E-mail: younhj@catholic.ac.kr
Background/Aims: Multiple myeloma (MM)-associated cardiac damage, particularly according to the type of monoclonal $(\mathrm{M})$ protein has not been elucidated. We sought to investigate relationship between elevated serum $M$ protein levels and echocardiographic indices of cardiac structure and function in patients with MM.

Methods: We evaluated a total of 184 consecutive MM patients who underwent echocardiography for bone marrow pre-transplant screening. Serum levels of intact immunoglobulin M protein and free light chain kappa/lambda (FLC- $\kappa /-\lambda$ ) were measured.

Results: One hundred thirty-nine patients were non-light chain MM(non-LCMM) and 45 patients belonged to LCMM. In patients with non-LCMM, significant correlations were found between serum $\mathrm{M}$ protein and left atrial volume index (LAVi; $r=0.720, p<0.0001), \mathrm{E} / \mathrm{e}^{\prime}(r=0.511, p<0.0001)$, and systolic pulmonary arterial pressure $(r=0.485, p<0.0001)$. In patients with LCMM, log-transformed FLC- $\lambda$ $(\log -\lambda)$ was correlated with left ventricular ejection fraction (LVEF, $r=-0.536, p=$ $0.010)$, left ventricular (LV) end-systolic dimension $(r=0.500, p=0.018)$, and LV end-systolic volume $(r=0.444, p=0.038)$. On multivariate analyses, hematocrit and serum $\mathrm{M}$ protein were independent predictors of LAVi in patients with non-LCMM. In patient with LCMM, FLC- $\lambda$ isotype was only found to be an independent determinant of LVEF.

Conclusions: An increase in serum M protein was associated with LV diastolic dysfunction, whereas an increase in serum FLC- $\lambda$ concentration showed a negative correlation with the echocardiographic parameters of LV systolic function. These findings also suggest that serum $\mathrm{M}$ protein has different effects on LV function according to the type of paraproteins in patients with MM.

Keywords: Multiple myeloma; Serum monoclonal paraprotein; Ventricular function, left

\section{INTRODUCTION}

Multiple myeloma (MM) is a B cell neoplasm characterized by clonal proliferation of malignant plasma cells in the bone marrow, and produces excess amounts of monoclonal protein (M protein) including an abnormal immunoglobulin (Ig) fragment or light chain that deposit in organs or tissues [1]. The toxic effects and organ 
dysfunction caused by $\mathrm{M}$ protein deposition differ in severity, clinical presentation, and prognosis from that caused by 'amyloidogenic' light chain deposition as seen in primary systemic amyloid light-chain (AL) amyloidosis [2]. In patients with AL amyloidosis, cardiac involvement is well known as the worst prognostic indicator [3]; however, there is not sufficient data regarding the relationship between elevated serum M protein levels and cardiac structure and function in patients with MM. Therefore, we sought to investigate the association of morphologic and functional echocardiographic features with the increased serum concentrations of $\mathrm{M}$ protein or free light chains (FLCs) in a population of MM patients undergoing autologous stem cell transplantation.

\section{METHODS}

\section{Study population}

We reviewed the medical records of 492 adults newly diagnosed with MM at a single institute from April 2003 to February 2013. Patients with primary AL amyloidosis were excluded for this study. Of these, 207 patients who underwent transthoracic Doppler echocardiography (TTE) for bone marrow pre-transplant screening constituted the initial study population after induction chemotherapy. Twenty-three of the 207 patients were excluded for the following reasons: moderate or greater degrees of valvular heart disease $(n=1)$, previous history of coronary artery disease $(\mathrm{n}=3$ ) or atrial fibrillation ( $\mathrm{n}$ $=4)$, connective tissue disease $(n=2)$, chronic obstructive pulmonary disease $(n=2)$, renal disease $(n=3)$, acute infection or sepsis $(n=4)$, and poor image quality $(n=$ 4). Patient clinical data were initially collected, and laboratory values including serum levels of $\mathrm{M}$ protein or FLCs were followed up at the time of echocardiographic examination after completion of induction chemotherapy. Approval for this study was obtained from the Seoul St. Mary's Hospital Institutional Review Board and the Catholic University of Korea in accordance with Declaration of Helsinki.

\section{Assays for serum M protein and FLC concentration}

Patients were assessed for symptomatic MM using the International Myeloma Working Group definition. Patients with non-light chain multiple myeloma (non-
LCMM) had a detectable M protein of intact Ig heavy chain type G, A, M, or D and either light chain kappa ( $\kappa)$ or lambda $(\lambda)$ [4]. Patients with LCMM who did not have intact IgM protein at diagnosis had measurable serum FLCs. The serum FLC- $\kappa$ and $-\lambda$ levels were measured using the FREELITE assay (The Binding Site, Birmingham, UK) and $\kappa / \lambda$ FLC ratios (FLC-R) were classified as nor$\mathrm{mal}$ (0.26 to 1.65) or abnormal (<0.26 or $>1.65$ ). Patients with $\kappa / \lambda$ FLC-R greater than 1.65 had excess $\kappa$-FLC and were presumed to be producing clonal $\kappa$-FLC. On the other hand, patients with ratios less than 0.26 had excess $\lambda$-FLC and were presumed to be producing $\lambda$-FLC [5].

\section{Echocardiography}

All ultrasound examinations were performed with a commercially available echocardiographic device (vivid 7, GE, Vingmed Ultrasound AS, Horten, Norway). Standard M-mode, two-dimensional and color Doppler measurements were obtained according to the American Society of Echocardiography recommendations [6]. Left ventricular (LV) volumes and ejection fraction (EF) were assessed by the biplane modified Simpson's method from apical four- and two-chamber views. The thickness of the interventricular septum and LV posterior wall and LV chamber dimensions were determined at end-diastole and end-systole from the M-mode at the level of chordae. LV mass was derived by the formula of Devereux and indexed to body surface area (left ventricular mass index [LVMi]). Left atrial (LA) volume was measured at end-systole using the biplane modified Simpson's rule in the apical four- and two-chamber views, and indexed to body surface area (left atrial volume index [LAVi]). Peak early (E) and late (A) diastolic flow velocities, and E-wave deceleration time (DT) were measured from a pulsed wave Doppler examination of the mitral inflow in the apical four-chamber view. The tissue Doppler-derived peak early (e') diastolic myocardial velocity measured at the septal and lateral mitral annulus was averaged, and as an index of LV filling pressure, E/e' was calculated. Left ventricular diastolic dysfunction (LVDD) was classified as normal, grade I (impaired relaxation), grade II (pseudonormal LV filling), or grade III (restrictive diastolic physiology) [7]. Right ventricular (RV) dimension and wall thickness were measured at end-diastole from the apical four chamber view and the subcostal view, respectively. RV function 
was assessed by RV descent, defined as the systolic displacement of the lateral portion of the tricuspid annular plane. Systolic pulmonary arterial pressure (SPAP) was estimated using the modified Bernoulli equation: SPAP $=4 \mathrm{~V}^{2}(\mathrm{v}=$ peak velocity of tricuspid regurgitation $)+$ estimated right atrial pressure [8]. All echocardiographic examinations were performed by skilled sonographers and interpreted by experienced cardiologists who blinded to the clinical information of the study population.

\section{Statistical methods}

Data are presented as mean \pm standard deviation $(\mathrm{SD})$, as medians with interquartile ranges (for biomarkers), or as counts and percentages. The Mann-Whitney U test or Student $t$ test was used to compare continuous variables between groups, and Pearson chi-square test or Fisher exact test was used to compare categorical variables between groups. Pearson or Spearman correlation coefficient was used to assess the linear relationship between two variables. Due to their skewed distribution, serum FLC- $\kappa$ (sFLC- $\kappa)$ and $-\lambda$ were log transformed. Multivariate regression analyses were performed to evaluate the independent predictors of left ventricular ejection fraction (LVEF) and LAVi, and for all analyses, a two-tailed $p$ $<0.05$ was considered statistically significant. Statistical analyses were performed using SPSS version 18 (SPSS Inc., Chicago, IL, USA).

\section{RESULTS}

\section{Baseline clinical characteristics and laboratory find- ings}

A total of 184 patients with MM were enrolled in this study. One hundred thirty-nine patients (76\%) had nonLCMM and 45 (24\%) had LCMM. Patient clinical characteristics are shown in Table 1. In the non-LCMM group, $95(68 \%)$ and $34(25 \%)$ of patients had detectable IgG and IgA, respectively, while only six (4\%) and four patients (3\%) had IgD and IgM, respectively. The proportion of patients with the sFLC- $\kappa$ isotype was higher than that of the sFLC- $\lambda$ isotype in patients with non-LCMM as well as LCMM, but the differences were not statistically significant. Both groups had an advanced Durie-Salmon stage III, and $\beta 2$-microglobulin and lactate dehydrogenase levels were similar between the non-LCMM and
LCMM subjects, whereas serum albumin levels were significantly decreased in non-LCMM when compared to LCMM patients at diagnosis. Patients with non-LCMM had lower serum creatinine levels, higher estimated glomerular filtration rate using the Modification of Diet in Renal Disease and higher erythrocyte sedimentation rate (ESR) levels than those with LCMM. sFLC- $\lambda$ levels were higher in LCMM patients, but sFLC- $\kappa$ and serum $\kappa / \lambda$ FLC-R did not differ significantly between the groups. Although the prevalence rate of smoking was higher in the LCMM group, there were no significant differences between these two groups in age, gender, body mass index, the prevalence of diabetes mellitus, hypertension (HTN), and hemoglobin ( $\mathrm{Hb}$ ), hematocrit (Hct), total cholesterol, and triglyceride levels. Also, there were no significant differences in chemotherapy regimens including high-dose dexamethasone between these two groups, and the maximum cumulative dose of doxorubicin that was used in vincristine, adriamycin and dexamethasone regimen did not exceed the limited cumulative dose in this population [9].

\section{Echocardiographic characteristics}

The time from diagnosis to TTE was similar between non-LCMM and LCMM patients $(3.3 \pm 2.8$ months vs. $3.4 \pm 2.8$ months, $p=0.768$ ). Patients with non-LCMM were more likely to have lower LVEF and larger left ventricular end-systolic diameter (LVESD) compared with LCMM patients. However, there were no significant differences in interventricular septal thickness, LV mass, LA volume, LV diastolic function, and RV chamber size, wall thickness, function and SPAP between the two groups (Table 2).

\section{Echocardiographic correlates of serum $M$ protein and log-transformed serum FLC- $\kappa$ and $-\lambda$}

We evaluated correlations between echocardiographic variables and serum levels of $\mathrm{M}$ protein, and log-transformed serum FLC- $\kappa /-\lambda(\log -\kappa /-\lambda)$ in patients with nonLCMM and LCMM, respectively (Table 3 ). In the nonLCMM group, serum M protein showed a weak positive association with left ventricular end-diastolic volume (LVEDV; $r=0.169, p=0.048)$, left ventricular end-systolic volume (LVESV; $r=0.230, p=0.007)$, and LVMi $(r=0.221$, $p=0.009)$. However, a significant positive correlation was found between serum M protein and LAVi $(r=0.720$, 
Table 1. Baseline clinical characteristics and laboratory findings of study population

\begin{tabular}{|c|c|c|c|}
\hline Variable & Non-LCMM $(\mathrm{n}=139)$ & $\operatorname{LCMM}(\mathrm{n}=45)$ & $p$ value \\
\hline Age, yr & $57 \cdot 7 \pm 9 \cdot 3$ & $56.3 \pm 8.4$ & 0.380 \\
\hline Male sex & $69(49.6)$ & $20(22.5)$ & 0.544 \\
\hline Body mass index, $\mathrm{kg} / \mathrm{m}^{2}$ & $23.6 \pm 2.9$ & $23.8 \pm 3.1$ & 0.657 \\
\hline Diabetes mellitus & $14(10.1)$ & $5(11.1)$ & 0.517 \\
\hline Hypertension & $39(28.1)$ & $14(31.1)$ & 0.694 \\
\hline Smoking & $10(7.2)$ & $9(20.0)$ & 0.019 \\
\hline Induction chemotherapy regimens & & & 0.661 \\
\hline Bortezomib & $60(43.1)$ & $24(53 \cdot 3)$ & \\
\hline Thalidomide & $37(26.6)$ & $12(26.6)$ & \\
\hline Bortezomib and thalidomide & $17(12.2)$ & $4(2.2)$ & \\
\hline Lenalidomide & $2(1.4)$ & $\mathrm{O}$ & \\
\hline High-dose dexamethasone & $23(16.5)$ & $5(11.1)$ & \\
\hline Ig heavy chain isotype, G/A/D/M & $95 / 34 / 6 / 4$ & - & \\
\hline Ig free light chain isotype & & & 0.449 \\
\hline к Type & $80(57.6)$ & $23(51.1)$ & \\
\hline$\lambda$ Type & $59(42.4)$ & $22(48.9)$ & \\
\hline Durie-Salmon stage $^{a}$ & & & 0.055 \\
\hline I & $3(2.2)$ & 0 & \\
\hline II & $18(13.0)$ & $1(2.2)$ & \\
\hline III & $117(84.8)$ & $44(97.8)$ & \\
\hline$\beta 2$-Microglobulin, mg/ $\mathrm{L}^{\mathrm{a}}$ & $3.5(2.6-6.4)$ & $5 \cdot 3(2.5-11.2)$ & 0.076 \\
\hline Albumin, $\mathrm{g} / \mathrm{dL}^{\mathrm{a}}$ & $3.2(2.8-3.7)$ & $3 \cdot 9(3 \cdot 5-4 \cdot 4)$ & $<0.0001$ \\
\hline Lactate dehydrogenase, $\mathrm{U} / \mathrm{L}^{\mathrm{a}}$ & $339(264-470)$ & $409(342-572)$ & 0.445 \\
\hline Hemoglobin, g/dL & $11.1(9.9-11.8)$ & $10.4(9.7-11.3)$ & 0.161 \\
\hline Hematocrit, \% & $33.1(29.7-35 \cdot 3)$ & $31.4(29.9-34.2)$ & 0.211 \\
\hline Serum creatinine, g/dL & $0.7(0.6-0.9)$ & $0.9(0.7-1.8)$ & 0.032 \\
\hline $\mathrm{eGFR}_{\mathrm{MDRD}}, \mathrm{mL} / \mathrm{min} / 1.73 \mathrm{~m}^{2}$ & $98.6(77 \cdot 6-120.6)$ & $69.9(32.6-110.9)$ & 0.021 \\
\hline $\mathrm{ESR}, \mathrm{mm} / \mathrm{hr}$ & $32.0(14.5-61.0)$ & $19.0(9.5-34.2)$ & 0.015 \\
\hline Total cholesterol, mg/dL & $170(145-202)$ & $159(135-189)$ & 0.404 \\
\hline Triglyceride, mg/dL & $131(85-181)$ & $130(101-185)$ & 0.680 \\
\hline Serum M protein, g/dL & $0.23(0.01-1.11)$ & - & \\
\hline Serum FLC-к, mg/dL & $15.8(9.0-31.3)$ & $21.1(10.3-87.4)$ & 0.066 \\
\hline Serum FLC- $\lambda$, mg/dL & $12.0(7.3-20.7)$ & $15.0(8.8-166.8)$ & 0.049 \\
\hline Serum $\kappa / \lambda$ FLC-R & $1.25(0.75-3.58)$ & $1.20(0.10-5.03)$ & 0.511 \\
\hline
\end{tabular}

Values are presented as mean $\pm \mathrm{SD}$, number (\%), or median (interquartile range).

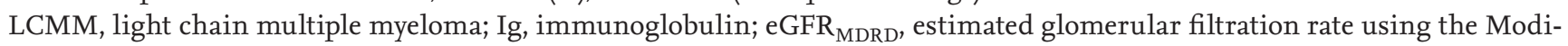
fication of Diet in Renal Disease; ESR, erythrocyte sedimentation rate; FLC, free light chain; FLC-R, free light chain ratio.

${ }^{\mathrm{a}}$ At diagnosis.

$p<0.0001)$, and serum $\mathrm{M}$ protein positively correlated with E/e' ratio $(r=0.511, p<0.0001)$. Serum M protein is also correlated significantly with $\operatorname{SPAP}(r=0.485, p<$
0.0001 ) (Fig. 1) and A' velocity $(r=0.321, p<0.0001)$. In LCMM patients with sFLC- $\kappa$ isotype, no echocardio-

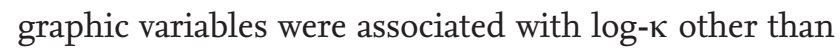


Table 2. Echocardiographic parameters of the study population

\begin{tabular}{|c|c|c|c|}
\hline Variable & Non-LCMM $(\mathrm{n}=139)$ & $\operatorname{LCMM}(\mathrm{n}=45)$ & $p$ value \\
\hline LVEF, \% & $61.1 \pm 5.1$ & $64.2 \pm 4.9$ & $<0.0001$ \\
\hline IVS, $\mathrm{mm}$ & $9.6 \pm 1.6$ & $9.8 \pm 2.1$ & 0.498 \\
\hline LVEDD, mm & $49.2 \pm 5.4$ & $47 \cdot 4 \pm 5 \cdot 4$ & 0.061 \\
\hline LVESD, mm & $30.7 \pm 5.0$ & $27 \cdot 9 \pm 5 \cdot 3$ & 0.002 \\
\hline LVEDV, mL & $79.8 \pm 24.9$ & $78.5 \pm 21.6$ & 0.748 \\
\hline LVESV, mL & $31.7 \pm 13.4$ & $28.6 \pm 11.0$ & 0.160 \\
\hline LVMi, g/m² & $105 \cdot 5 \pm 24 \cdot 5$ & $107.4 \pm 37.9$ & 0.745 \\
\hline $\mathrm{LAVi}, \mathrm{mL} / \mathrm{m}^{2}$ & $29.1 \pm 10.9$ & $29.6 \pm 11.7$ & 0.804 \\
\hline $\mathrm{E}, \mathrm{cm} / \mathrm{sec}$ & $63.0 \pm 18.4$ & $66.8 \pm 20.9$ & 0.244 \\
\hline $\mathrm{A}, \mathrm{cm} / \mathrm{sec}$ & $76.1 \pm 18.8$ & $73.7 \pm 19.1$ & 0.462 \\
\hline $\mathrm{E} / \mathrm{A}$ & $0.8 \pm 0.3$ & $0.9 \pm 0.4$ & 0.129 \\
\hline DT, msec & $214 \pm 48$ & $212 \pm 52$ & 0.761 \\
\hline $\mathrm{E} / \mathrm{e}^{\prime}$ & $9.4 \pm 3.1$ & $10.3 \pm 3.7$ & 0.133 \\
\hline $\mathrm{A}^{\prime}, \mathrm{cm} / \mathrm{sec}$ & $9.9 \pm 2.7$ & $9.9 \pm 2.1$ & 0.900 \\
\hline LVDD grade & & & 0.057 \\
\hline Normal & $20(14.8)$ & $11(25 \cdot 6)$ & \\
\hline GI & $112(83.0)$ & $29(67.4)$ & \\
\hline GII & $3(2.2)$ & $3(7.0)$ & \\
\hline RVDd, mm & $32.7(30.2-34.9)$ & $32.4(29.4-34.5)$ & 0.696 \\
\hline RVWT, mm & $3.9(3.4-4.5)$ & $4.1(3.5-4.7)$ & 0.241 \\
\hline TAPSE, mm & $22.8 \pm 3.8$ & $23 \cdot 0 \pm 4 \cdot 3$ & 0.918 \\
\hline SPAP, $\mathrm{mmHg}$ & $27.1 \pm 6.9$ & $29.7 \pm 10.6$ & 0.157 \\
\hline
\end{tabular}

Values are presented as mean $\pm \mathrm{SD}$, number (\%), or median (interquartile range).

LCMM, light chain multiple myeloma; LVEF, left ventricular ejection fraction; IVS, interventricular septal thickness; LVEDD, left ventricular end-diastolic diameter; LVESD, left ventricular end-systolic diameter; LVEDV, left ventricular end-diastolic volume; LVESV, left ventricular end-systolic volume; LVMi, left ventricular mass index; LAVi, left atrial volume index; DT, deceleration time; E/e', peak early diastolic transmitral velocity to peak early diastolic mitral annular velocity; LVDD, left ventricular diastolic dysfunction; RVDd, right ventricular end-diastolic diameter; RVWT, right ventricular wall thickness; TAPSE, tricuspid annular plane systolic excursion; SPAP, systolic pulmonary artery pressure.

SPAP $(r=0.378, p=0.025)$ as shown in Table 3. On the other hand, in the sFLC- $\lambda$ isotype group, log- $\lambda$ showed a statistically significant negative correlation with LVEF $(r=-0.536, p=0.010$ ) (Fig. 2), and positively correlated with LVESD $(r=0.500, p=0.018)$ and LVESV $(r=0.444, p$ $=0.038)$. Although the low levels of sFLC- $\kappa$ and $-\lambda$ were detected in patients with non-LCMM, they did not show any associations with echocardiographic parameters.

It may be controversial whether decreased serum M protein levels after induction chemotherapy can reflect echocardiographic findings or not, because initial serum levels of $\mathrm{M}$ protein and FLC- $\kappa, \lambda$ showed a significant decrease after induction chemotherapy in both groups (Supplementary Table 1). Therefore, we reanalyzed the associations between initial serum $M$ protein levels and echocardiographic parameters of LV function. Interestingly, initial serum $\mathrm{M}$ protein showed weaker correlations with LAVi $(r=0.202, p=0.023), E / e^{\prime}$ ratio $(r=0.252, p=0.005)$, and $\operatorname{SPAP}(r=0.220, p=0.022)$ in non-LCMM, whereas there was a stronger relationship between initial log- $\lambda$ and $\operatorname{LVEF}(r=-0.668, p=0.001)$ in LCMM (Supplementary Table 2).

\section{Independent predictors of left ventricular systolic and diastolic function}

In patients with non-LCMM, Hct $(\beta,-0.353$; 95\% confi- 
Table 3. Correlation between echocardiographic parameters and serum levels of monoclonal protein and log-transformed serum free light chains in the study population

\begin{tabular}{|c|c|c|c|c|c|c|}
\hline \multirow{3}{*}{ Variable } & \multirow{2}{*}{\multicolumn{2}{|c|}{$\frac{\text { Non-LCMM }}{\text { M protein }(\mathrm{n}=139)}$}} & \multicolumn{4}{|c|}{ LCMM } \\
\hline & & & \multicolumn{2}{|c|}{$\log -\kappa(n=23)$} & \multicolumn{2}{|c|}{$\log -\lambda(n=22)$} \\
\hline & $r$ & $p$ value & $r$ & $p$ value & $r$ & $p$ value \\
\hline LVEF, \% & -0.130 & - & 0.383 & - & -0.536 & 0.010 \\
\hline IVS, mm & 0.164 & - & 0.214 & - & 0.301 & - \\
\hline LVEDD, mm & 0.038 & - & 0.111 & - & 0.318 & - \\
\hline LVESD, mm & 0.095 & - & 0.079 & - & 0.500 & 0.018 \\
\hline LVEDV, mL & 0.169 & 0.048 & 0.143 & - & 0.301 & - \\
\hline LVESV, mL & 0.230 & 0.007 & 0.059 & - & 0.444 & 0.038 \\
\hline LVMi, g/m² & 0.221 & 0.009 & 0.194 & - & 0.378 & - \\
\hline $\mathrm{LAVi}, \mathrm{mL} / \mathrm{m}^{2}$ & 0.720 & $<0.0001$ & 0.170 & - & 0.366 & - \\
\hline $\mathrm{E}, \mathrm{cm} / \mathrm{sec}$ & 0.132 & - & 0.265 & - & 0.414 & - \\
\hline $\mathrm{A}, \mathrm{cm} / \mathrm{ec}$ & 0.321 & $<0.0001$ & 0.077 & - & 0.256 & - \\
\hline $\mathrm{E} / \mathrm{A}$ & -0.169 & - & 0.237 & - & 0.310 & - \\
\hline DT, msec & 0.036 & - & -0.402 & - & -0.043 & - \\
\hline $\mathrm{E} / \mathrm{e}^{\prime}$ & 0.511 & $<0.0001$ & 0.200 & - & 0.042 & - \\
\hline $\mathrm{A}^{\prime}, \mathrm{cm} / \mathrm{sec}$ & 0.067 & - & -0.218 & - & -0.137 & - \\
\hline RVDd, mm & 0.021 & - & 0.218 & - & 0.111 & - \\
\hline RVWT, mm & 0.051 & - & 0.128 & - & 0.223 & - \\
\hline TAPSE, mm & -0.005 & - & 0.071 & - & -0.231 & - \\
\hline SPAP, mmHg & 0.485 & $<0.0001$ & 0.378 & 0.025 & 0.242 & - \\
\hline
\end{tabular}

LCMM, light chain multiple myeloma; Log- $\kappa$, log-transformed serum level of kappa; Log- $\lambda$, log-transformed serum level of lambda; LVEF, left ventricular ejection fraction; IVS, interventricular septal thickness; LVEDD, left ventricular end-diastolic diameter; LVESD, left ventricular end-systolic diameter; LVEDV, left ventricular end-diastolic volume; LVESV, left ventricular end-systolic volume; LVMi, left ventricular mass index; LAVi, left atrial volume index; DT, deceleration time; E/e', peak early diastolic transmitral velocity to peak early diastolic mitral annular velocity; RVDd, right ventricular end-diastolic diameter; RVWT, right ventricular wall thickness; TAPSE, tricuspid annular plane systolic excursion; SPAP, systolic pulmonary artery pressure.
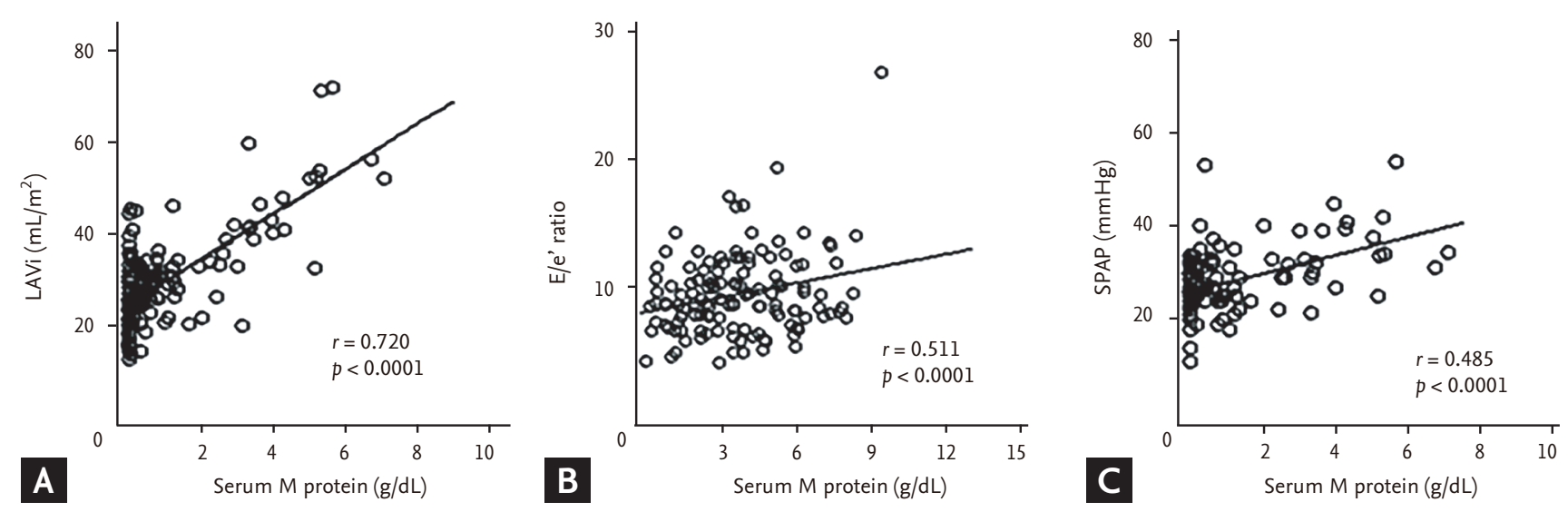

Figure 1. Correlation between serum monoclonal (M) protein and left atrial volume index (LAVi), peak early diastolic transmitral velocity to peak early diastolic mitral annular velocity (E/e'), or systolic pulmonary artery pressure (SPAP) in non-light chain multiple myeloma patients. Serum M protein showed a significant positive correlation with (A) LAVi, (B) E/e', or (C) SPAP. 

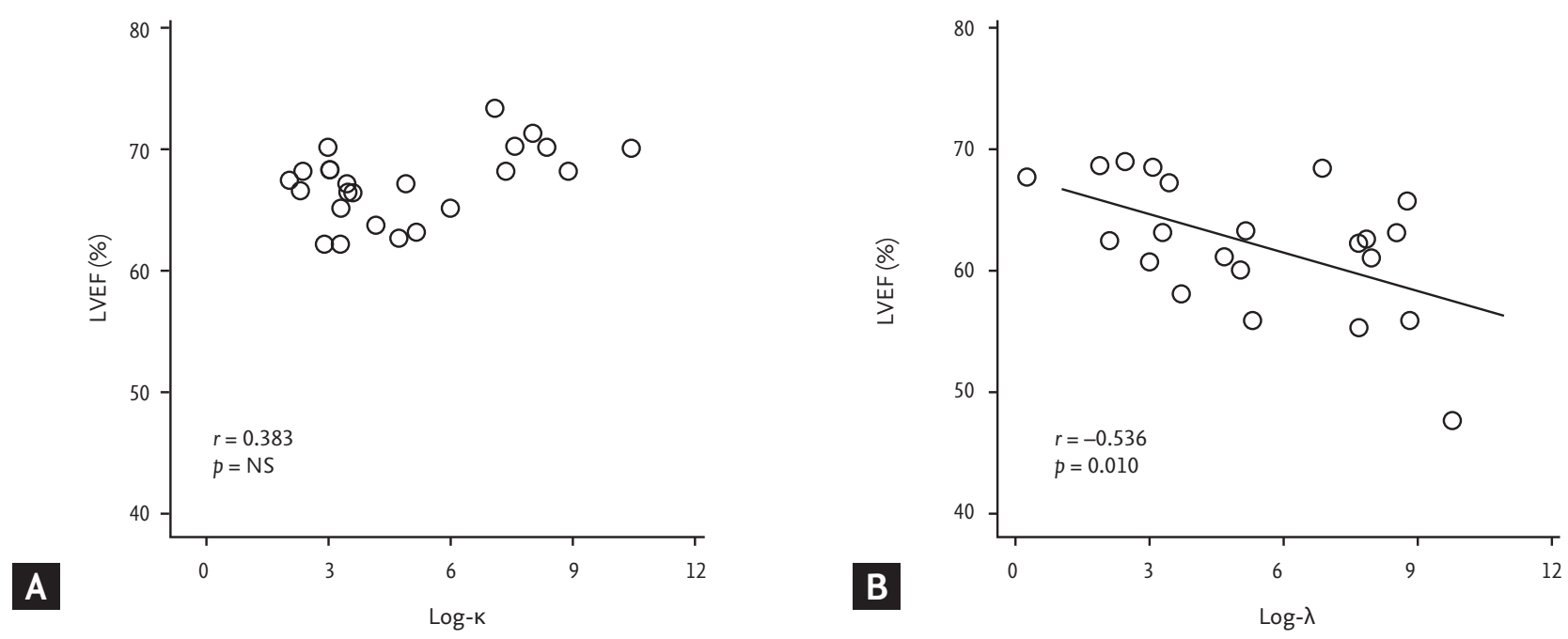

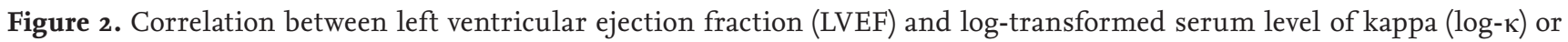
log-transformed serum level of lambda $(\log -\lambda)$ in light chain multiple myeloma patients. (A) Log- $\kappa$ showed no significant association with LVEF in patients with $\kappa$-isotype $(n=23)$. On the other hand, $(B) \log -\lambda$ showed a statistically significant negative correlation with LVEF in patients with $\lambda$-isotype $(n=22)$. NS, not significant.

dence interval [CI], -0.666 to $-0.040 ; p=0.027)$ and serum M protein ( $\beta, 4.304 ; 95 \% \mathrm{CI}, 3.404$ to $5.204 ; p<0.0001)$ were found to be significant independent determinants of LAVi after adjusting for potential confounders such as age, HTN, LV diastolic function and use of bortezomib. On the other hand, multivariate analysis for LVEF after adjusting $\mathrm{HTN}, \mathrm{Hb}$, serum $\mathrm{M}$ protein, sFLC isotype and use of bortezomib demonstrated that $\operatorname{ESR}(\beta,-0.035 ; 95 \%$ CI, -0.063 to $-0.007 ; p=0.013$ ) was an independent predictor of LVEF. In patients with LCMM, sFLC- $\lambda$ isotype $(\beta,-5.340 ; 95 \% \mathrm{CI},-7.870$ to $-2.810 ; p<0.0001)$ was an independent predictor of LVEF after adjusting for other parameters including age, HTN, and use of bortezomib. However, multivariate analysis for LAVi after adjusting age, HTN, use of bortezomib and sFLC- $\lambda$ isotype revealed that Hct $(\beta,-0.959 ; 95 \% \mathrm{CI},-1.773$ to $-0.144 ; p=$ 0.022) was only found to be an independent predictor of LAVi.

\section{DISCUSSION}

To the best of our knowledge, this is the first study to demonstrate echocardiographic correlates of the increased serum concentrations of M protein or FLCs in patients with MM. The main findings of the current in- vestigation are as follows: (1) in the non-LCMM group, increased serum $M$ protein was significantly correlated with echocardiographic parameters of LV diastolic function, and Hct and serum $M$ protein contributed to the prediction of LAVi; (2) in the LCMM group, patients with the sFLC- $\lambda$ isotype showed significant association between log- $\lambda$ and echocardiographic parameters related to LV systolic function, and sFLC- $\lambda$ isotype contributed to the prediction of LVEF.

\section{Non-LCMM}

Previous studies have reported that monoclonal hypergammoglobulinemia increases serum viscosity, resulting in increase of blood flow resistance combined with expanded plasma volume, and this condition can lead to systemic hypertension, congestive heart failure $(\mathrm{CHF})$, and disturbance of peripheral circulation $[10,11]$. Although no patients presented with symptomatic hyperviscosity in this study, we found significant positive correlations between serum M protein and LAVi, E/e' ratio, and SPAP, which are echocardiographic variables reflecting LV filling pressure.

LA enlargement has been proposed as an indicator of diastolic burden and a predictor of adverse cardiovascular outcomes such as atrial fibrillation, stroke, and CHF [12]. The E/e' ratio has been also used as a tool for assess- 
ing LV filling pressures; however, this Doppler index may be more affected by loading conditions, especially in healthy subjects with preserved EF $[13,14]$. Considering the clinical conditions of our patients who received frequent blood transfusion or hydration, and that they showed preserved LV systolic function $(\mathrm{LVEF}>50 \%)$, the $\mathrm{E} / \mathrm{e}^{\prime}$ ratio might not have expressed accurate LV filling pressure, which is the reason why we used LAVi as a surrogate marker of LV diastolic function on multivariate analysis. Nevertheless, the significant relation between serum $\mathrm{M}$ protein and $\mathrm{E} / \mathrm{e}$ ' ratio as well as LAVi strongly suggests that the increase in serum $M$ protein is associated with LVDD.

Symptoms of hyperviscosity have been reported to occur when levels reach $5 \mathrm{~g} / \mathrm{dL}$ for IgG, $7 \mathrm{~g} / \mathrm{dL}$ for IgA, and $4 \mathrm{~g} / \mathrm{dL}$ for IgM; however, little is known about how levels of serum $\mathrm{M}$ protein might predict subclinical hemodynamic changes. Somer [15] described that not only an increase in paraprotein concentration, but also their molecular characteristics can contribute to increase of serum viscosity. Earlier experimental studies demonstrated that fibrinogen, a major plasma protein, promotes red blood cell (RBC) aggregation by interaction with erythrocyte membranes, resulting in increase of blood viscosity, and this process usually occurs to a great extent in MM patients with dysfibrinogenemia $[16,17]$. Furthermore, recently published laboratory studies documented that these hemorheological changes can affect on blood flow resistance via a variety of vascular control mechanisms [18]. In previous reviews of the literatures, Hct has been described as an important determinant of whole blood viscosity, and ESR, a non-specific inflammatory marker, has been also reported to reflect the degree of RBC aggregation [19].

Consistent with aforementioned studies, our results showed that Hct and serum M protein were significant predictors of LAVi. Unexpectedly, Hct showed an inverse relationship with LAVi in both univariate and multivariate analyses, but this result may be due to RBC transfusion or recombinant erythropoietin use for anemia correction.

\section{LCMM}

Monoclonal light chain deposits in the myocardium are well known in patients with AL amyloidosis or light chain deposition disease, causing a restrictive infiltra- tive cardiomyopathy $[20,21]$. On the other hand, cardiac involvement by non-amyloidotic light chain in MM patients without amyloidosis has only been described in few case reports [22,23]. Although Shustik et al. [24] documented that the group with $\lambda$-light chain disease (LCD) had a shorter median survival than the $\kappa$-LCD group (10 months vs. 30 months, $p<0.0001$ ), they could not find any significant differences between the two groups in prognostic factors.

In the present study focusing on echocardiographic characteristics of MM, an increase in SFLC- $\lambda$ was significantly associated with a decrease in LVEF, and sFLC- $\lambda$ isotype was found to be an independent predictor of LVEF. Ig FLCs are approximately $22 \mathrm{kDa}$ polypeptides, and they may exist as monomers or as dimers with a molecular weight of approximately $44 \mathrm{kDa}$ that is covalently or noncovalently disulfide-linked. FLC- $\kappa$ are usually monomers, and thus they are filtered three-times faster than FLC- $\lambda$ that exist as dimers, which is the reason why the serum concentration of FLC- $\kappa$ is lower than that of FLC- $\lambda$ despite the higher production of $\kappa$ light chain in comparison with $\lambda$ light chain [25]. Therefore, this physiologic characteristic of sFLC- $\lambda$ that has a relatively long half-life in the blood may contribute the possibility of $\lambda$ light chain deposition in the myocardium. In this study, the presence of FLCs in the myocardium could not be confirmed through endomyocardial biopsy. However, given the results of previously reported literatures, our findings strongly suggest that the sFLC (especially the sFLC- $\lambda$ ) might be deposited in the myocardium.

Our investigation has several limitations due to its cross-sectional design. First, our patients did not have available initial echocardiographic data, and moreover, induction chemotherapy could affect LV function. Second, FLCs deposition was not confirmed by endomyocardial biopsy, and hemodynamic data was not also verified through right heart catheterization. However, risks related to these invasive procedures limit their routine use in clinical practice. Third, few participants showed significant LV systolic dysfunction (LVEF < 56\%, $\mathrm{n}=17,9.2 \%$ ) as we enrolled patients in relatively good condition who were preparing for autologous stem cell transplantation. Nonetheless, LVDD ( $\geq$ grade I) or LA enlargement was found in more than half of the study population, which is clinically important, because in many previous reports, LVDD has been shown to prog- 
ress to CHF with preserved LVEF [26]. Fourth, newer imaging modalities such as speckle tracking echocardiography or cardiac magnetic resonance imaging were not performed, because typical echocardiographic findings suggestive of severe left ventricular hypertrophy or cardiac dysfunction were not found among study population. Finally, blood viscosity could not be directly measured, and cardiac biomarkers such as N-terminal pro-brain natriuretic peptide or cardiac troponin I were not assessed in this study.

In conclusion, serum $\mathrm{M}$ protein has different effects on cardiac function according to the type of paraproteins in patients with MM, not AL amyloidosis. Increase in serum $\mathrm{M}$ protein, including an abnormal Ig fragment or light chain is associated with LVDD, raising the possibility of hemodynamic abnormality caused by increased viscosity in patients with non-LCMM. On the other hand, increase of sFLC- $\lambda$ concentration shows a negative correlation with echocardiographic indices of LV systolic function, suggesting that its deposition may lead to myocardial damage in patients with LCMM. These results suggest that assessment of serum M protein levels can provide useful information about hemodynamic condition and may be helpful to determine treatment choice or drug dose before bone marrow transplantation in patients with MM. The findings of this retrospective study should be validated in the future prospective studies enrolling larger patient populations and with serial echocardiography follow-up.

\section{KEY MESSAGE}

1. An increase in serum monoclonal (M) protein was associated with left ventricular (LV) diastolic dysfunction, and an increase in serum free light chain- $\lambda$ showed an inverse relationship with LV systolic function among patients with multiple myeloma (MM).

2. Serum M protein has different effects on LV function according to the type of paraprotein in patients with MM.

\section{Conflict of interest}

No potential conflict of interest relevant to this article was reported.

\section{Acknowledgments}

This study was supported by grant of the Korea Health Technology R\&D Project, Ministry of Health and Welfare, Republic of Korea (A120175).

\section{REFERENCES}

1. Kyle RA, Rajkumar SV. Multiple myeloma. N Engl J Med 2004;351:1860-1873.

2. Rajkumar SV, Gertz MA, Kyle RA. Primary systemic amyloidosis with delayed progression to multiple myeloma. Cancer 1998;82:1501-1505.

3. Cueto-Garcia L, Reeder GS, Kyle RA, et al. Echocardiographic findings in systemic amyloidosis: spectrum of cardiac involvement and relation to survival. J Am Coll Cardiol 1985;6:737-743.

4. International Myeloma Working Group. Criteria for the classification of monoclonal gammopathies, multiple myeloma and related disorders: a report of the International Myeloma Working Group. Br J Haematol 2003;121:749-757.

5. Dispenzieri A, Kyle R, Merlini G, et al. International Myeloma Working Group guidelines for serum-free light chain analysis in multiple myeloma and related disorders. Leukemia 2009;23:215-224.

6. Lang RM, Bierig M, Devereux RB, et al. Recommendations for chamber quantification: a report from the American Society of Echocardiography's Guidelines and Standards Committee and the Chamber Quantification Writing Group, developed in conjunction with the European Association of Echocardiography, a branch of the European Society of Cardiology. J Am Soc Echocardiogr 2005;18:1440-1463.

7. Nagueh SF, Appleton CP, Gillebert TC, et al. Recommendations for the evaluation of left ventricular diastolic function by echocardiography. J Am Soc Echocardiogr 2009;22:107-133.

8. Rudski LG, Lai WW, Afilalo J, et al. Guidelines for the echocardiographic assessment of the right heart in adults: a report from the American Society of Echocardiography endorsed by the European Association of Echocardiography, a registered branch of the European Society of Cardiology, and the Canadian Society of Echocardiography. J Am Soc Echocardiogr 2010;23:685-713.

9. Swain SM, Whaley FS, Ewer MS. Congestive heart failure 
in patients treated with doxorubicin: a retrospective analysis of three trials. Cancer 2003;97:2869-2879.

10. Russell JA, Powles RL. The relationship between serum viscosity, hypervolaemia and clinical manifestations associated with circulating paraprotein. Br J Haematol 1978;39:163-175.

11. Mehta J, Singhal S. Hyperviscosity syndrome in plasma cell dyscrasias. Semin Thromb Hemost 2003;29:467-471.

12. Abhayaratna WP, Seward JB, Appleton CP, et al. Left atrial size: physiologic determinants and clinical applications. J Am Coll Cardiol 2006;47:2357-2363.

13. Ommen SR, Nishimura RA, Appleton CP, et al. Clinical utility of Doppler echocardiography and tissue Doppler imaging in the estimation of left ventricular filling pressures: a comparative simultaneous Doppler-catheterization study. Circulation 2000;102:1788-1794.

14. Firstenberg MS, Levine BD, Garcia MJ, et al. Relationship of echocardiographic indices to pulmonary capillary wedge pressures in healthy volunteers. J Am Coll Cardiol 2000;36:1664-1669.

15. Somer T. Rheology of paraproteinaemias and the plasma hyperviscosity syndrome. Baillieres Clin Haematol 1987;1:695-723.

16. Carr ME Jr, Zekert SL. Abnormal clot retraction, altered fibrin structure, and normal platelet function in multiple myeloma. Am J Physiol 1994;266(3 Pt 2):H1195-H1201.

17. Morsdorf S, Jung F, Seyfert UT, Mrowietz C, Pindur G, Wenzel E. Haemostatical and rheological aspects of dysfibrinogenemia. Clin Hemorheol Microcirc 1997;17:13-19.
18. Baskurt OK, Yalcin O, Meiselman HJ. Hemorheology and vascular control mechanisms. Clin Hemorheol Microcirc 2004;30:169-178.

19. Baskurt OK, Meiselman HJ. Blood rheology and hemodynamics. Semin Thromb Hemost 2003;29:435-450.

20. Liao R, Jain M, Teller P, et al. Infusion of light chains from patients with cardiac amyloidosis causes diastolic dysfunction in isolated mouse hearts. Circulation 2001;104:1594-1597.

21. Jimenez-Zepeda VH. Light chain deposition disease: novel biological insights and treatment advances. Int J Lab Hematol 2012;34:347-355.

22. Schattner A, Epstein M, Berrebi A, Caspi A. Case report: multiple myeloma presenting as a diastolic heart failure with no evidence of amyloidosis. Am J Med Sci 1995;310:256-257.

23. Garton MJ, Walton S, Ewen SW. Systemic lambda lightchain deposition presenting with predominant cardiac involvement. Postgrad Med J 1993;69:588-591.

24. Shustik C, Bergsagel DE, Pruzanski W. Kappa and lambda light chain disease: survival rates and clinical manifestations. Blood 1976;48:41-51.

25. Ozsan GH, Dispenzieri A. Serum free light chain analysis in multiple myeloma and plasma cell dyscrasias. Expert Rev Clin Immunol 2011;7:65-73.

26. Wan SH, Vogel MW, Chen HH. Pre-clinical diastolic dysfunction. J Am Coll Cardiol 2014;63:407-416.

artery pressure. 